\title{
NEARLY KÄHLER MANIFOLDS WITH POSITIVE HOLOMORPHIC SECTIONAL CURVATURE
}

\author{
By Kouei Sekigawa and Takuji Sato
}

\section{§1. Introduction.}

An almost Hermitian manifold $(M, J,\langle\rangle$,$) is called a nearly Kähler manifold$ provided that $\left(\nabla_{X} J\right) Y+\left(\nabla_{Y} J\right) X=0$ for all $X, Y \in \mathscr{X}(M)(\mathscr{X}(M)$ denotes the Lie algebra of all smooth vector fields on $M$ ). From the definition, it follows immediately that a Kähler manifold is necessarily a nearly Kähler manifold. In the present paper, we shall study the structure of nearly Kähler manifolds with positive holomorphic sectional curvature. In $\S 2$, we recall some elementary formulas in a nearly Kähler manifold. In $\S 3$, we establish an integral formula on the unit sphere bundle over a compact Einstein nearly Kähler manifold. In $\S 4$, we discuss the pinching problem on the holomorphic sectional curvature of a compact non-Kähler, nearly Kähler manifold and show some results related to the ones obtained by Tanno [18], Takamatsu and the second named author [17].

In [7], Gray studied the structure of positively curved compact nearly Kähler manifolds and proposed the following conjecture :

Conjecture : Let $M=(M, J,\langle\rangle$,$) be a compact nearly Kähler manifold with$ positive sectional curvature. If the scalar curvature of $M$ is constant, then $M$ is isometric to a complex projective space with a Kähler metric of constant holomorphic sectional curvature or a 6-dimensional sphere with a Riemannian metric of constant sectional curvature.

For Kähler manifolds, this conjecture is positive (cf. [5], [10], etc.). However, for non-Kähler, nearly Kähler manifolds, this conjecture is negative. Namely, we shall give a counter example to this conjecture in the last section.

The authors wish to express their hearty thanks to the referee who pointed out some errors in the original manuscript.

\section{§2. Preliminaries.}

In this section, we prepare some elementary formulas in a nearly Kähler manifold. Let $M=(M, J,\langle\rangle$,$) be an n(=2 m)$-dimensional connected nearly Kähler manifold. We denote by $\nabla$ and $R$ the Riemannian connection and the curvature tensor of $M$, respectively. We assume that the curvature tensor $R$ is defined by

Received December 21, 1983 


$$
R(X, Y) Z=\nabla_{[X, Y]} Z-\left[\nabla_{X}, \nabla_{Y}\right] Z, \quad X, Y, Z \in \mathscr{X}(M) .
$$

We denote by $R_{1}$ and $R_{1}^{*}$ the Ricci tensor and the Ricci *-tensor of $M$, respectively. The tensor field $R_{1}$ and $R_{1}^{*}$ are defined respectively by

$$
R_{1}(x, y)=\text { Trace of }(z \mapsto R(x, z) y) \text {, }
$$

and

$$
R_{1}^{*}(x, y)=(1 / 2) \text { Trace of }(z \mapsto R(J y, x) J z),
$$

for $x, y, z \in M_{p}$ (the tangent space of $M$ at $p$ ) (cf. [9], [19]). Then it is known that the tensor fields $R_{1}$ and $R_{1}^{*}$ satisfy the following equalities:

$$
\begin{array}{ll}
R_{1}(X, Y)=R_{1}(Y, X), & R_{1}(J X, J Y)=R_{1}(X, Y), \\
R_{1}^{*}(X, Y)=R_{1}^{*}(Y, X), & R_{1}^{*}(J X, J Y)=R_{1}^{*}(X, Y),
\end{array}
$$

for $X, Y \in \mathscr{X}(M)$. The first Chern form $\gamma$ of $M$ is given by

$$
8 \pi \gamma(X, Y)=5 R_{1}^{*}(J X, Y)-R_{1}(J X, Y),
$$

for all $X, Y \in \mathscr{X}(M)$ ([9], p. 238).

We denote by $S$ the scalar curvature of $M$. The sectional curvature, the holomorphic sectional curvature and the holomorphic bisectional curvature are defined respectively by

$$
K(x, y)=\frac{\langle R(x, y) x, y\rangle}{\|x\|^{2}\|y\|^{2}},
$$

for $x, y \in M_{p}(p \in M)$ with $x \neq 0, y \neq 0,\langle x, y\rangle=0$,

$$
H(x)=K(x, J x),
$$

for $x \in M_{p}(p \in M)$ with $x \neq 0$, and

$$
B(x, y)=\frac{\langle R(x, J x) y, J y\rangle}{\|x\|^{2}\|y\|^{2}},
$$

for $x, y \in M_{p}(p \in M)$ with $x \neq 0, y \neq 0$.

A nearly Kähler manifold $M$ is said to be of holomorphically $\delta$-pinched $(0 \leqq \delta \leqq 1)$ if there exists a positive constant $l$ such that

$$
\delta l \leqq H(x) \leqq l,
$$

for all non-zero $x \in M_{p}$, for all $p \in M$. Since we are dealing with nearly Kähler manifolds, the size $\left\|\left(\nabla_{x} J\right) y\right\|^{2}$ will be important in the pinching estimates. A nearly Kähler manifold $M$ is said to satisfy the condition $T(\rho, \sigma)$ if

$$
\rho H(x) \leqq\left\|\left(\nabla_{x} J\right) y\right\|^{2} \leqq \sigma H(x),
$$


for $x, y \in M_{p}$ with $\|x\|=\|y\|=1,\langle x, y\rangle=\langle x, J y\rangle=0$ for all $p \in M$ ([7]]).

In the present paper, we shall adopt the following notational convention. For an orthonormal basis $\left\{e_{i}\right\}=\left\{e_{\alpha}, e_{m+\alpha}=J e_{\alpha}\right\}(1 \leqq \alpha, \beta, \cdots \leqq m ; 1 \leqq a, b, \cdots \imath, \jmath, k$, $\cdots \leqq n=2 m)$, of $M_{p}(p \in M)$, we put

$$
\begin{gathered}
\left.e_{\bar{\imath}}=J e_{\imath} \quad \text { (and hence } e_{\bar{\alpha}}=e_{m+\alpha}, e_{\overline{m+\alpha}}=-e_{\alpha}\right), \\
R_{h \imath j k}=\left\langle R\left(e_{h}, e_{\imath}\right) e_{\jmath}, e_{k}\right\rangle, \quad R_{\bar{h} \imath j k}=\left\langle R\left(e_{\bar{h}}, e_{\imath}\right) e_{\jmath}, e_{k}\right\rangle, \\
\cdots, R_{\bar{h} \bar{j} \bar{k}}=\left\langle R\left(e_{\bar{h}}, e_{\bar{\imath}}\right) e_{\bar{\imath}}, e_{\bar{k}}\right\rangle, \\
\nabla_{l} R_{h \imath j k}=\left\langle\left(\nabla_{e_{l}} R\right)\left(e_{h}, e_{\imath}\right) e_{\jmath}, e_{k}\right\rangle, \nabla_{\bar{l}} R_{h \imath j k}=\left\langle\left(\nabla_{e_{\bar{l}}} R\right)\left(e_{h}, e_{\imath}\right) e_{\jmath}, e_{k}\right\rangle, \\
\cdots, \nabla_{\bar{l}} R_{\bar{h} \bar{j} \bar{j} \bar{k}}=\left\langle\left(\nabla_{\bar{l}} R\right)\left(e_{\bar{h}}, e_{\bar{\imath}}\right) e_{\bar{j}}, e_{\bar{k}}\right\rangle, \text { etc., }
\end{gathered}
$$

and

$$
R_{\imath \jmath}=R_{1}\left(e_{\imath}, e_{j}\right), \quad R_{\imath j}^{*}=R_{1}^{*}\left(e_{\imath}, e_{\jmath}\right) .
$$

The following equalities in $M$ are well-known ([7], [9], etc.):

$$
\begin{aligned}
\langle R(w, x) y, z\rangle- & \langle R(w, x) J y, J z\rangle=\left\langle\left(\nabla_{w} J\right) x,\left(\nabla_{y} J\right) z\right\rangle, \\
\langle R(w, x) y, z\rangle=\langle R(J w, J x) J y, J z\rangle & \\
\left\langle\left(\nabla^{2} e_{e_{i} e_{\jmath}} J\right) x, y\right\rangle= & \frac{1}{2}\left(\left\langle R\left(e_{\imath}, J e_{\jmath}\right) x, y\right\rangle-\left\langle R\left(J y, e_{\imath}\right) e_{\jmath}, x\right\rangle\right. \\
& \left.+\left\langle R\left(J x, e_{\imath}\right) e_{\jmath}, y\right\rangle\right),
\end{aligned}
$$

$$
\left\|\nabla R_{1}-\nabla R_{1}^{*}\right\|^{2}=(1 / 8) \text { Trace of }\left\{\left(R^{1}-\left(R^{*}\right)^{1}\right) \circ\left(R^{1}-5\left(R^{*}\right)^{1}\right) \circ\left(R^{1}-\left(R^{*}\right)^{1}\right)\right\},
$$

where $\left\langle R^{1} x, y\right\rangle=R_{1}(x, y),\left\langle\left(R^{*}\right)^{1} x, y\right\rangle=R_{1}^{*}(x, y), w, x, y, z \in M_{p} \quad(p \in M)$. Вy (2.2), (2.3) and (2.18), we have

$$
\sum_{i=1}^{n}\left\langle\left(\nabla^{2}{ }_{e_{i} e_{i}} J\right) x, y\right\rangle=R_{1}^{*}(J x, y)-R_{1}(J x, y),
$$

for $x, y \in M_{p}(p \in M)$. By (2.2), (2.3) and (2.16), we have

$$
\sum_{i=1}^{n}\left\langle\left(\nabla_{e_{i}} J\right) x,\left(\nabla_{e_{i}} J\right) y\right\rangle=R_{1}(x, y)-R_{1}^{*}(x, y),
$$

for $x, y \in M_{p}$. By (2.3), (2.4), (2.5), (2.16) and (2.21), we have

$$
\begin{aligned}
& \sum_{a=1}^{n} R_{a \bar{a} \imath \bar{\jmath}}=2 R_{\imath \jmath}^{*}, \\
& \sum_{a=1}^{n} R_{a \bar{j} j \bar{a}}=R_{\imath \jmath}^{*} .
\end{aligned}
$$

We note that $\left\langle\left(\nabla_{x} J\right) y, z\right\rangle\left(x, y, z \in M_{p}\right)$ satisfies the followings: 


$$
\left\langle\left(\nabla_{x} J\right) y, z\right\rangle=-\left\langle\left(\nabla_{y} J\right) x, z\right\rangle=-\left\langle\left(\nabla_{x} J\right) z, y\right\rangle,
$$

and

$$
\left\langle\left(\nabla_{J x} J\right) J y, z\right\rangle=-\left\langle\left(\nabla_{x} J\right) y, z\right\rangle .
$$

By (2.7), (2.8), (2.9) and (2.16), we have

$$
\begin{gathered}
K(x, y)=(1 / 8)\{3 H(x+J y)+3 H(x-J y)-H(x+y)-H(x-y) \\
-H(x)-H(y)\}+(3 / 4)\left\|\left(\nabla_{x} J\right) y\right\|^{2}, \\
B(x, y)=K(x, y)+K(x, J y)-2\left\|\left(\nabla_{x} J\right) y\right\|^{2},
\end{gathered}
$$

for $x, y \in M_{p}(p \in M)$ with $\|x\|=\|y\|=1,\langle x, y\rangle=\langle x, J y\rangle=0$.

\section{§3. An integral formula on the unit sphere bundle.}

The following fact is well-known and useful for our arguments ([2]) :

Proposition 3.1. Let $\boldsymbol{R}^{n}$ be an n-dimensional Euclidean space and $f$ a homogeneous polynomial of degree $r(\geqq 1)$ defined on $\boldsymbol{R}^{n}$. Then we have

$$
\int_{S n-1(1)}(D f) \omega_{2}=r(n+r-2) \int_{S^{n-1(1)}}\left(\left.f\right|_{S n-1(1)}\right) \omega_{2},
$$

where $D$ denotes the Laplace operator of $\boldsymbol{R}^{n}$ and $\omega_{2}$ denotes the volume element of an $(n-1)$-dimensional unit sphere $S^{n-1}(1)$ with the canonical Riemannian metric.

Let $M=(M,\langle\rangle$,$) be an n$-dimensional connected Riemannian manifold. We denote by $T(M)$ and $S(M)$ the tangent bundle and the unit sphere bundle over $M$, respectively :

$$
\begin{aligned}
& T(M)=\left\{(p, x) \mid p \in M, x \in M_{p}\right\}, \\
& S(M)=\{(p, x) \in T(M) \mid\|x\|=1\} .
\end{aligned}
$$

For each point $p \in M$, we put

$$
S_{p}=\left\{x \in M_{p} \mid\|x\|=1\right\} .
$$

Then $S_{p}$ is isometric to $S^{n-1}(1)$. We now recall the Sasaki metric $\langle,\rangle^{s}$ on $T(M)$ (cf. [12]). We denote by $X^{h}$ (resp. $X^{v}$ ) the horizontal lift (resp. the vertical lift) of $X \in \mathscr{X}(M)$. Then the Sasaki metric $\langle,\rangle^{s}$ on $T(M)$ is defined by

$$
\left\langle X^{h}, Y^{h}\right\rangle^{s}=\langle X, Y\rangle,\left\langle X^{v}, Y^{v}\right\rangle^{s}=\langle X, Y\rangle,\left\langle X^{h}, Y^{v}\right\rangle^{s}=0,
$$

for $X, Y \in \mathfrak{X}(M)$. From (3.1), we get easily

$$
\left(\nabla_{X} Y^{h}\right)_{(p, x)}=\left(\nabla_{X} Y\right)^{h}+\frac{1}{2}(R(X, Y) x)^{v},
$$


where $\nabla^{s}$ denotes the Riemannian connection on $T(M)$ with respect to the Sasaki metric $\langle,\rangle^{s}$. From (3.2), we see that any horizontal lift of a geodesic in $M$ is a geodesic in $T(M)=\left(T(M),\langle,\rangle^{s}\right)$. We denote by using the same notation $\langle,\rangle^{s}$ the induced metric on $S(M)$ which is induced from the Sasaki metric $\langle,\rangle^{s}$ on $T(M)$. Let $\omega$ (resp. $\omega_{1}$ ) be the volume element on $S(M)$ (resp. $M$ ) with respect to the metric $\langle,\rangle^{s}$ (resp. $\left.\langle\rangle,\right)$. Then we have easily

$$
\omega(p, x)=\omega_{1}(p) \wedge \omega_{2}(x), \quad(p, x) \in S(M) .
$$

If $M$ is compact and orientable, by (3.3), for any smooth function $f$ on $S(M)$, we have

$$
\int_{S(M)} f \omega=\int_{M}\left\{\int_{S_{p}} f(p, x) \omega_{2}(x)\right\} \omega_{1}(p)
$$

Let $(p, x)$ be any point of $S(M)$. We take an orthonormal basis $\left\{e_{i}\right\}=$ $\left\{e_{1}, \cdots, e_{n}\right\}$ of $M_{p}$ such that $x=e_{1}$. Then $\left\{e_{1}{ }^{n}, \cdots, e_{n}{ }^{h}, e_{2}{ }^{v}, \cdots, e_{n}{ }^{v}\right\}$ is an orthonormal basis of the tangent space $S(M)_{(p, x)}$. For each $y \in M_{p}$, the tangent space $\left(M_{p}\right)_{y}$ (i.e., the vertical subspace of $\left.T(M)_{(p, y)}\right)$ is identified with $M_{p}$ by means of parallel translation. Under this identification, $e_{2}{ }^{v}$ corresponds to $e_{2}$ $(1 \leqq i \leqq n)$. We denote by $\left(u_{1}, \cdots, u_{n}, v_{2}, \cdots, v_{n}\right)$ the normal coordinate system on a neighborhood of $(p, x)$ in $S(M)$ with respect to the orthonormal basis $\left\{e_{1}{ }^{h}\right.$, $\left.\cdots, e_{n}{ }^{h}, e_{2}{ }^{v}, \cdots, e_{n}{ }^{v}\right\}$. In [10], Gray has introduced a second order linear differential operator $L$ by

$$
L_{(p, x)}=\left\{\sum_{\imath=1}^{n} \frac{\partial^{2}}{\partial u_{\imath}^{2}}+\frac{1}{2} \sum_{\imath, j \geq 2} h_{\imath j} \frac{\partial^{2}}{\partial v_{i} \partial v_{\jmath}}\right\}_{(p, x)},
$$

where $h_{\imath j}(p, x)=\left\langle R\left(e_{\imath}, x\right) e_{\jmath}, x\right\rangle$. We denote by $\Delta^{h}$ the horizontal Laplacian of $S(M)$. Then in terms of the normal coordinate system $\left(u_{1}, \cdots, u_{n}, v_{2}, \cdots, v_{n}\right)$, $\Delta^{h}$ is given by

$$
\Delta_{(p, x)}^{h}=\left\{\sum_{\imath=1}^{n} \frac{\partial^{2}}{\partial u_{\imath}^{2}}\right\}_{(p, x)} .
$$

For a smooth function $f$ on $S(M)$, we denote by $\operatorname{grad}^{h} f\left(\operatorname{resp} . \operatorname{grad}^{v} f\right.$ ) the horizontal (resp. the vertical) component of $\operatorname{grad} f$.

Now, let $M=(M, J,\langle\rangle$,$) be an n(=2 m)$-dimensional nearly Kähler manifold. We may regard holomorphic sectional curvature $H=H(x)$ as a smooth function on $S(M)$. Then we have

$$
\begin{aligned}
\left(\operatorname{grad}^{n} H\right)_{(p, x)} & =\sum_{\imath=1}^{n}\left\{\left\langle\left(\nabla_{e_{i}} R\right)(x, J x) x, J x\right\rangle+2\left\langle R(x, J x) x,\left(\nabla_{e_{i}} J\right) x\right\rangle\right\} e_{\imath}{ }^{n}, \\
\left(\operatorname{grad}^{v} H\right)_{(p, x)} & =(\operatorname{grad} H)_{(p, x)}-\left(\operatorname{grad}^{n} H\right)_{(p, x)}-\left\langle(\operatorname{grad} H)_{(p, x)}, x^{v}\right\rangle^{s} x^{v} \\
& =4 \sum_{\imath=2}^{n}\left\langle R(x, J x) x, J e_{\imath}\right\rangle e_{\imath}{ }^{v} .
\end{aligned}
$$

By (3.8), we see that 


$$
\left\langle\left(\operatorname{grad}^{v} H\right)_{(p, x)}, x^{v}\right\rangle^{s}=\left\langle\left(\operatorname{grad}^{v} H\right)_{(p, x)},(J x)^{v}\right\rangle^{s}=0 .
$$

From the result due to Tanno [18] and (3.8), we may note the following

Proposition 3.2. Let $M=(M, J,\langle\rangle$,$) be a nearly Kähler manifold. Then$ $M$ is a space of constant holomorphic sectional curvature if and only if $\operatorname{grad}^{v} H=0$ on $S(M)$.

We assume that $M=(M, J,\langle\rangle$,$) is a connected compact Einstein nearly$ Kähler manifold. First, we estimate the value $L(H)(p, x)$ at any point $(p, x) \in S(M)$. By (3.6) and (3.7), we get

$$
\begin{aligned}
\sum_{i=1}^{n} \frac{\partial^{2} H}{\partial u_{\imath}^{2}}(p, x)=\left(\Delta^{h} H\right)(p, x) \\
=\sum_{i=1}^{n}\left\langle\nabla_{e_{i}}^{s}\left(\operatorname{grad}^{h} H\right), e_{i}{ }^{h}\right\rangle \\
=\sum_{i=1}^{n}\left\{\left\langle\left(\nabla_{e_{i} e_{i}}^{2} R\right)(x, J x) x, J x\right\rangle+\left\langle\left(\nabla_{e_{i}} R\right)\left(x,\left(\nabla_{e_{i}} J\right) x\right) x, J x\right\rangle\right. \\
\quad+\left\langle\left\langle\nabla_{e_{i}} R\right)(x, J x) x,\left(\nabla_{e_{i}} J\right) x\right\rangle+2\left\langle\left(\nabla_{e_{i}} R\right)(x, J x) x,\left(\nabla_{e_{i}} J\right) x\right\rangle \\
\left.\quad+2\left\langle R\left(x,\left(\nabla_{e_{i}} J\right) x\right) x,\left(\nabla_{e_{i}} J\right) x\right\rangle+2\left\langle R(x, J x) x,\left(\nabla_{e_{i} e_{i}}^{2} J\right) x\right\rangle\right\} \\
=\sum_{i=1}^{n}\left\{\left\langle\left(\nabla_{e_{i} e_{i}}^{2} R\right)(x, J x) x, J x\right\rangle+4\left\langle\left(\nabla_{e_{i}} R\right)(x, J x) x,\left(\nabla_{e_{i}} J\right) x\right\rangle\right. \\
\left.\quad+2\left\langle R\left(x,\left(\nabla_{e_{i}} J\right) x\right) x,\left(\nabla_{e_{i}} J\right) x\right\rangle+2\left\langle R(x, J x) x,\left(\nabla_{e_{i} e_{i}}^{2} J\right) x\right\rangle\right\} .
\end{aligned}
$$

Taking account of the first Bianchi, the second Bianchi and the Ricci identities, and (2.16), (2.17), (2.20), we get

$$
\begin{aligned}
& \frac{1}{2} \sum_{\imath=1}^{n}\left\langle\left(\nabla_{e_{i} e_{i}}^{2} R\right)(x, J x) x, J x\right\rangle \\
& =\frac{1}{2} \sum_{\imath=1}^{n}\left\{\left\langle\left(\nabla_{e_{\imath} x}^{2} R\right)\left(e_{\imath}, J x\right) x, J x\right\rangle-\left\langle\left(\nabla_{e_{i} J x}^{2} R\right)\left(e_{\imath}, x\right) x, J x\right\rangle\right\} \\
& =\frac{1}{2}\left\{\sum_{\imath=1}^{n}\left\langle\left(\nabla_{x e_{i}}^{2} R\right)\left(e_{\imath}, J x\right) x, J x\right\rangle\right. \\
& \quad+\sum_{\imath, j=1}^{n}\left(R_{\imath x \imath j} R_{j \bar{x} x \bar{x}}+R_{\imath x \bar{x} j} R_{\imath \jmath x \bar{x}}+R_{\imath x x j} R_{i \bar{x} j \bar{x}}+R_{\imath x \bar{x} j} R_{i \bar{x} x j}\right) \\
& \quad-\sum_{\imath=1}^{n}\left\langle\left(\nabla_{J x e_{i}}^{2} R\right)\left(e_{\imath}, x\right) x, J x\right\rangle \\
& \left.\quad-\sum_{\imath, j=1}^{n}\left(R_{i \bar{x} \imath \jmath} R_{\jmath x x \bar{x}}+R_{i \bar{x} x j} R_{\imath \jmath x \bar{x}}+R_{i \bar{x} x j} R_{\imath x j \bar{x}}+R_{i \bar{x} \bar{x} j} R_{\imath x x j}\right)\right\} \\
& =\sum_{\imath, j=1}^{n}\left\{R_{\imath x \jmath x} \delta_{\imath j} H(x)-R_{\imath x \jmath x} R_{i \bar{x} j \bar{x}}-R_{\imath x \bar{x} j}\left(R_{\imath x \bar{x} j}+2 R_{i \bar{x} \jmath x}\right)\right\}
\end{aligned}
$$




$$
\begin{aligned}
& =\sum_{\imath, \jmath=1}^{n} R_{\imath x \jmath x}\left(\delta_{i j} H(x)-R_{i \bar{x} j \bar{x}}\right) \\
& +\sum_{\imath, j=1}^{n} R_{\imath x j \bar{x}}\left(-R_{\imath x x \bar{j}}+\left\langle\left(\nabla_{e_{i}} J\right) x,\left(\nabla_{J x} J\right) e_{\jmath}\right\rangle+2 R_{i \bar{x} \bar{j} \bar{x}}\right. \\
& \left.+2\left\langle\left(\nabla_{e_{i}} J\right) J x,\left(\nabla_{e_{j}} J\right) x\right\rangle\right) \\
& =\sum_{\imath, j=1}^{n}\left\{R_{\imath x j x}\left(\delta_{i j} H(x)-R_{i \bar{x} j \bar{x}}\right)\right. \\
& +\left(-R_{\imath x j x}+\left\langle\left(\nabla_{e_{i}} J\right) x,\left(\nabla_{e_{j}} J\right) x\right\rangle\right)\left(R_{\imath x \jmath x}+2 R_{i \bar{x} j \bar{x}}\right. \\
& \left.\left.-3\left\langle\left(\nabla_{e_{i}} J\right) x,\left(\nabla_{e_{j}} J\right) x\right\rangle\right)\right\} \\
& =\sum_{\imath, \jmath=1}^{n} R_{\imath x \jmath x}\left\{\delta_{i j} H(x)-R_{\imath x \jmath x}-3 R_{i \bar{x} j \bar{x}}+3\left\langle\left(\nabla_{e_{i}} J\right) x,\left(\nabla_{e_{j}} J\right) x\right\rangle\right\} \\
& +3 \sum_{\imath, \jmath=1}^{n} R_{\imath x \bar{j} \bar{x}}\left\langle\left(\nabla_{e_{i}} J\right) x,\left(\nabla_{e_{j}} J\right) x\right\rangle,
\end{aligned}
$$

where we put $R_{\imath x \jmath x}=R_{i 1 j 1}, R_{i \bar{x} j \bar{x}}=R_{i 1 j 1}, \cdots$, etc. Thus, by (3.9) and (3.10), we have

$$
\begin{aligned}
& \sum_{\imath=1}^{n} \frac{\partial^{2} H}{\partial u_{\imath}^{2}}(p, x) \\
& =2\left\{\sum_{\imath, j=1,}^{n} R_{\imath x j x}\left(\delta_{i j} H(x)-R_{\imath x \jmath x}-3 R_{i \bar{x} j \bar{x}}+3\left\langle\left(\nabla_{e_{i}} J\right) x,\left(\nabla_{e_{j}} J\right) x\right\rangle\right)\right. \\
& \left.\quad+3 \sum_{\imath, j=1}^{n} R_{\imath x \bar{j} \bar{x}}\left\langle\left(\nabla_{e_{i}} J\right) x,\left(\nabla_{e_{j}} J\right) x\right\rangle\right\} \\
& \quad+4 \sum_{i=1}^{n}\left\langle\left(\nabla_{e_{i}} R\right)(x, J x) x,\left(\nabla_{e_{i}} J\right) x\right\rangle+2 \sum_{i=1}^{n}\left\langle R\left(x,\left(\nabla_{e_{i}} J\right) x\right) x,\left(\nabla_{e_{i}} J\right) x\right\rangle \\
& \quad+2 \sum_{i=1}^{n}\left\langle R(x, J x) x,\left(\nabla_{e_{i} e_{i}}^{2} J\right) x\right\rangle .
\end{aligned}
$$

Similarly, we have

$$
\frac{\partial^{2} H}{\partial v_{i} \partial v_{\jmath}}(p, x)=-4\left\{\delta_{i \jmath} H(x)-R_{\imath x_{\jmath} x}-3 R_{i \bar{x} j \bar{x}}+3\left\langle\left(\nabla_{e_{i}} J\right) x,\left(\nabla_{e_{\jmath}} J\right) x\right\rangle\right\} .
$$

We now define smooth functions $f_{\lambda}(\lambda=1,2,3,4)$ on $S(M)$ by

$$
\begin{aligned}
& f_{1}(p, x)=\sum_{\imath, j=1}^{n} R_{\imath x \bar{j} \bar{x}}\left\langle\left(\nabla_{e_{i}} J\right) x,\left(\nabla_{e_{j}} J\right) x\right\rangle, \\
& f_{2}(p, x)=\sum_{i=1}^{n}\left\langle\left(\nabla_{e_{i}} R\right)(x, J x) x,\left(\nabla_{e_{i}} J\right) x\right\rangle, \\
& f_{3}(p, x)=\sum_{i=1}^{n}\left\langle R\left(x,\left(\nabla_{e_{i}} J\right) x\right) x,\left(\nabla_{e_{i}} J\right) x\right\rangle,
\end{aligned}
$$




$$
\begin{aligned}
f_{4}(\not, x) & =\sum_{i=1}^{n}\left\langle R(x, J x) x,\left(\nabla_{e_{i} e_{i}}^{2} J\right) x\right\rangle \\
& =-\left\langle R(x, J x) x,\left(R^{1}-\left(R^{*}\right)^{1}\right) J x\right\rangle .
\end{aligned}
$$

From (3.5), (3.11), (3.12) and (3.13), we have

$$
L(H)(p, x)=6 f_{1}(p, x)+4 f_{2}(p, x)+2 f_{3}(p, x)+2 f_{4}(p, x),
$$

for all $(p, x) \in S(M)$. Since $M$ is an Einstein space, it follows that the operator $L$ is self-adjoint (cf. [10]). Thus, we have the following equality ([10], p. 42):

$$
\begin{aligned}
0 & =\int_{S(M)} L\left(H^{2}\right) \omega \\
& =\int_{S(M)}\left\{2 H L(H)+2\left\|\operatorname{grad}^{h} H\right\|^{2}+\left\langle R\left(x, \operatorname{grad}^{v} H\right) x, \operatorname{grad}^{v} H\right\rangle\right\} \omega .
\end{aligned}
$$

We shall evaluate the integral $\int_{S(M)}\left\|\operatorname{grad}^{h} H\right\|^{2} \omega$. We define smooth functions $g_{\mu}(\mu=1,2,3)$ on $S(M)$ by

$$
\begin{aligned}
& g_{1}(p, x)=\sum_{i=1}^{n}\left\langle\left(\nabla_{e_{i}} R\right)(x, J x) x, J x\right\rangle^{2}, \\
& g_{2}(p, x)=\sum_{i=1}^{n}\left\langle\left(\nabla_{e_{i}} R\right)(x, J x) x, J x\right\rangle\left\langle R(x, J x) x,\left(\nabla_{e_{i}} J\right) x\right\rangle, \\
& g_{3}(p, x)=\sum_{i=1}^{n}\left\langle R(x, J x) x,\left(\nabla_{e_{i}} J\right) x\right\rangle^{2},
\end{aligned}
$$

for $(p, x) \in S(M)$. Then, by (3.7) and (3.16), we get

$$
\int_{S(M)}\left\|\operatorname{grad}^{h} H\right\|^{2} \omega=\int_{S(M)} g_{1} \omega+4 \int_{S(M)}\left(g_{2}+g_{3}\right) \omega .
$$

Taking account of (3.4), (3.13), (3.16), Proposition 3.1 and Green's theorem, we have

$$
\int_{S(M)} g_{2} \omega=-2 \int_{S(M)} g_{3} \omega-\int_{S(M)} H\left(f_{2}+f_{3}+f_{4}\right) \omega .
$$

From the results due to Gray [8] and the second named author [13], we may note that $M$ is a Riemannian locally 3-symmetric space if and only if $g_{1}$ is identically zero.

By (3.14), (3.15), (3.17) and (3.18), we have finally

$$
\int_{S(M)}\left[2\left\{g_{1}-4 g_{3}+H\left(6 f_{1}-2 f_{3}-2 f_{4}\right)\right\}+\left\langle R\left(x, \operatorname{grad}^{v} H\right) x, \operatorname{grad}^{v} H\right\rangle\right] \omega=0 .
$$

The integral formula (3.19) together with (3.13) and (3.16) plays an important role in the arguments of the next section.

In the rest of this section, we assume that $M=(M, J,\langle\rangle$,$) is a connected$ 
non-Kähler, Einstein nearly Kähler manifold with vanishing first Chern form (i. e., $R_{1}=5 R_{1}^{*}$ ). By making use of (2.22), (3.8) and Proposition 3.1, we have the followings:

$$
\begin{gathered}
\int_{S_{p}} H \omega_{2}=\frac{8 S}{5 n(n+2)} V_{2}, \\
\int_{S_{p}} H^{2} \omega_{2}=\frac{1}{4(n+2)} \int_{S_{p}}\left\|\operatorname{grad}^{v} H\right\|^{2} \omega_{2}+\frac{64 S^{2}}{25 n^{2}(n+2)^{2}} V_{2},
\end{gathered}
$$

where $V_{2}=\operatorname{Vol}\left(S^{n-1}(1)\right)$. By (3.8) and (3.21), we have

$$
\begin{aligned}
\int_{S_{p}} \sum_{k=1}^{n}\left(R_{x \bar{x} x k}\right)^{2} \omega_{2} & =\frac{1}{16} \int_{S_{p}}\left\|\operatorname{grad}^{v} H\right\|^{2} \omega_{2}+\int_{S_{p}} H^{2} \omega_{2} \\
& =\frac{n+6}{16(n+2)} \int_{S_{p}}\left\|\operatorname{grad}^{v} H\right\|^{2} \omega_{2}+\frac{64 S^{2}}{25 n^{2}(n+2)^{2}} V_{2} .
\end{aligned}
$$

If $M$ is holomorphically $\delta$-pinched, by (2.10) and (3.20), we get

$$
\delta l \leqq \frac{8 S}{5 n(n+2)} \leqq l
$$

\section{$\S 4$. Some results.}

S. Tanno [18] has proved the following

PROPOSITION 4.1. If a 6-dimensional nearly Kähler manifold $M=(M, J,\langle\rangle$, is of constant holomorphic sectional curvature $H$, then either $M$ is Kählerian, or $M$ is of constant sectional curvature $H>0$.

First, in connection with the above result, we shall show some results. Let $M=(M, J,\langle\rangle$,$) be a 6$-dimensional connected non-Kähler, nearly Kähler manifold. Then it is known that $M$ is an Einstein space with positive scalar curvature and vanishing first Chern form (i.e., $R_{1}=5 R_{1}^{*}$ ), and furthermore the following equalities hold $([11])$ :

$$
\begin{gathered}
\left\langle\left(\nabla_{e_{h}} J\right) e_{\imath},\left(\nabla_{e_{j}} J\right) e_{k}\right\rangle=-\frac{S}{30}\left\{\left\langle e_{\imath}, e_{\jmath}\right\rangle\left\langle e_{h}, e_{k}\right\rangle-\left\langle e_{h}, e_{\jmath}\right\rangle\left\langle e_{\imath}, e_{k}\right\rangle\right. \\
\left.-\left\langle J e_{\imath}, e_{\jmath}\right\rangle\left\langle J e_{h}, e_{k}\right\rangle+\left\langle J e_{h}, e_{\jmath}\right\rangle\left\langle J e_{\imath}, e_{k}\right\rangle\right\}, \\
\left\langle\left(\nabla_{e_{k} e_{j}}^{2} J\right) e_{2}, e_{h}\right\rangle=-\frac{S}{30}\left\{\left\langle e_{k}, e_{\jmath}\right\rangle\left\langle J e_{\imath}, e_{h}\right\rangle+\left\langle e_{k}, e_{\imath}\right\rangle\left\langle J e_{h}, e_{\jmath}\right\rangle\right. \\
\left.+\left\langle e_{k}, e_{h}\right\rangle\left\langle J e_{j}, e_{\imath}\right\rangle\right\},
\end{gathered}
$$

where $\left\{e_{i}\right\}=\left\{e_{\alpha}, e_{3+\alpha}=J e_{\alpha}\right\} \quad(\alpha=1,2,3)$ is an orthonormal basis of $M_{p}(p \in M)$.

We now evaluate the values $f_{\lambda}(p, x)(\lambda=1,3,4)$. By (2.22), (3.13) and (4.1), we get 


$$
\begin{aligned}
& f_{1}(p, x)=\frac{S}{30}\left(\frac{S}{30}-H(x)\right), \\
& f_{3}(p, x)=-\frac{S}{30}\left(H(x)-\frac{S}{6}\right) .
\end{aligned}
$$

Since $M$ is an Einstein space with $R_{1}=5 R_{1}^{*}$, by (3.13), we get

$$
f_{4}(p, x)=-\frac{2 S}{15} H(x) \text {. }
$$

By (3.16), (3.22) and (4.1), we get

$$
\int_{S_{p}} g_{3} \omega_{2}=\frac{S}{480} \int_{S_{p}}\left\|\operatorname{grad}^{v} H\right\|^{2} \omega_{2} .
$$

THEOREM 4.2. Let $M=(M, J,\langle\rangle$,$) be a 6-dimensional connected complete$ non-Kähler, nearly Kähler manifold satisfying the condition

$$
K(x, y)>\frac{S}{120},
$$

for $x, y \in M_{p}$ with $\|x\|=\|y\|=1,\langle x, y\rangle=\langle x, J y\rangle=0$, for all $p \in M$. Then $M$ is isometric to a 6-dimensional sphere of constant curvature $S / 30$.

Proof. Since $M$ is an Einstein space with positive scalar curvature, $M$ is compact by Myer's theorem. By (3.19) (3.21), (4.3) (4.6), we have

$$
\int_{S(M)}\left\{2 g_{1}+\left\langle R\left(x, \operatorname{grad}^{v} H\right) x, \operatorname{grad}^{v} H\right\rangle-\frac{S}{120}\left\|\operatorname{grad}^{v} H\right\|^{2}\right\} \omega=0 .
$$

From the hypothesis, (4.7) and Proposition 4.1, the theorem follows immediately.

Q.E.D.

Furthermore, we have the following

THEOREM 4.3. Let $M=(M, J,\langle\rangle$,$) be a 6-dimensional connected complete$ non-Kähler, nearly Kähler manifold. If $M$ is holomorphically $\delta(>2 / 5)$-pinched, then $M$ is isometric to a 6-dimensional sphere of constant curvature $S / 30$.

Proof. By the hypothesis and (2.10), (2.24), (3.23) and (4.1), we have

$$
\begin{aligned}
K(x, y) & \geqq \frac{1}{4}(3 \delta-2) l+\frac{S}{40} \\
& >-\frac{1}{5} l+\frac{S}{40} \\
& >-\frac{S}{60}+\frac{S}{40}=\frac{S}{120},
\end{aligned}
$$

for $x, y \in M_{p}$ with $\|x\|=\|y\|=1,\langle x, y\rangle=\langle x, J y\rangle=0$, for all $p \in M$. Thus the 
theorem follows immediately from Theorem 4.2.

Q.E.D.

Next, we shall deal with general cases where the dimension of $M$ is not necessarily equal to 6 . In connection with the results obtained by Bishop and Goldberg ([3], [4], [5]), we have the following

THEOREM 4.4. Let $M=(M, J,\langle\rangle$,$) be an n(=2 m)$-dimensional connected compact non-Kähler, nearly Kähler manifold with constant scalar curvature. If $M$ satisfies the condition

$$
K(x, y)+K(x, J y)+B(x, y)>0,
$$

for $x, y \in M_{p}$ with $x \neq 0, y \neq 0,\langle x, y\rangle=\langle x, J y\rangle=0$, for all $p \in M$, then the Riccr tensor $R_{1}$ of $M$ is parallel and the first Chern form of $M$ vanishes.

Proof. Since $M$ is compact and the scalar curvature $S$ of $M$ is constant, by the result due to Tachibana [16], the first Chern form $\gamma$ is a harmonic 2-form.

For each point $p \in M$, we may choose an orthonormal basis $\left\{e_{i}\right\}=\left\{e_{\alpha}, e_{\bar{\alpha}}\right\}$ which diagonalizes the symmetric linear endomorphism $5\left(R^{*}\right)^{1}-R^{1}$ of $M_{p}$. By the choice of $\left\{e_{i}\right\}$, we get

$$
\gamma\left(e_{2}, e_{j}\right)=0 \text { for } e_{j} \neq \pm e_{i} .
$$

For the 2 -form $\gamma$, we put

$$
F(\gamma)=\sum_{\imath, j, k} R_{\imath j} \gamma_{i k} \gamma_{j k}-\frac{1}{2} \sum_{h, \imath, j, k} R_{h \imath j k} \gamma_{h \imath} \gamma_{j k},
$$

where $\gamma_{\imath j}=\gamma\left(e_{\imath}, e_{j}\right)$. By (4.9), (4.10) reduces to

$$
F(\gamma)=2 \sum_{\alpha, \beta}\left\{\left(R_{\alpha \beta \alpha \beta}+R_{\alpha \bar{\beta} \alpha \bar{\beta}}\right)\left(\gamma_{\alpha \bar{\alpha}}\right)^{2}-R_{\alpha \bar{\alpha} \beta \bar{\beta}} \gamma_{\alpha \bar{\alpha} \bar{\alpha}} \gamma_{\beta \bar{\beta}}\right\} .
$$

By (2.25) and (4.11), we get

$$
F(\gamma)=2 \sum_{\alpha<\beta}\left\{R_{\alpha \bar{\alpha} \beta \bar{\beta}}\left(\gamma_{\alpha \bar{\alpha}}-\gamma_{\beta \bar{\beta}}\right)^{2}+2\left\|\left(\nabla_{e_{\alpha}} J\right) e_{\beta}\right\|^{2}\left(\gamma_{\alpha \bar{\alpha}}^{2}+\gamma_{\beta \bar{\beta}}^{2}\right)\right\},
$$

or

$$
F(\gamma)=2 \sum_{\alpha<\beta}\left\{\left(R_{\alpha \beta \alpha \beta}+R_{\alpha \bar{\beta} \alpha \bar{\beta}}\right)\left(\gamma_{\alpha \bar{\alpha}}-\gamma_{\beta \bar{\beta}}\right)^{2}+4\left\|\left(\nabla_{e_{\alpha}} J\right) e_{\beta}\right\|^{2} \gamma_{\alpha \bar{\alpha}} \gamma_{\beta \bar{\beta}}\right\} .
$$

By (4.12), we have finally

$$
\begin{aligned}
F(\gamma)=\sum_{\alpha<\beta}\left\{\left(R_{\alpha \bar{\alpha} \beta \bar{\beta}}+R_{\alpha \beta \alpha \beta}+\right.\right. & \left.R_{\alpha \bar{\beta} \alpha \bar{\beta}}\right)\left(\gamma_{\alpha \bar{\alpha}}-\gamma_{\beta \bar{\beta}}\right)^{2} \\
& \left.+2\left\|\left(\nabla_{e_{\alpha}} J\right) e_{\beta}\right\|^{2}\left(\gamma_{\alpha \bar{\alpha}}+\gamma_{\beta \bar{\beta}}\right)^{2}\right\} .
\end{aligned}
$$

Since $\gamma$ is a harmonic 2-form and $F(\gamma) \geqq 0$, according to Yano and Bochner [22], it follows that $F(\gamma)=0$ and $\gamma$ is parallel. Thus, by (4.12) and (4.13), we get

$$
\gamma_{\alpha \bar{\alpha}}-\gamma_{\beta \bar{\beta}}=0 \text {, and }\left\|\left(\nabla_{e_{\alpha}} J\right) e_{\beta}\right\|^{2}\left(\gamma_{\alpha \bar{\alpha}}+\gamma_{\beta \bar{\beta}}\right)^{2}=0 \text {, }
$$


for $1 \leqq \alpha<\beta \leqq m$.

Since $M$ is non-Kählerian, it follows that

$$
\left(\nabla_{e_{\alpha}} J\right) e_{\beta} \neq 0 \quad \text { for some } \alpha<\beta .
$$

Thus, by (4.14) and (4.15), we have

$$
\left.\gamma=0 \quad \text { (i. e. } R_{1}=5 R_{1}^{*}\right) .
$$

Therefore, by (2.19) and (4.16), we have

$$
\nabla\left(R_{1}-R_{1}^{*}\right)=0
$$

and hence

$$
\nabla R_{1}=0 \text {. }
$$

Q.E.D.

Furthermore, we have the following

THEOREM 4.5. Let $M=(M, J,\langle\rangle$,$) be an n(=2 m)$-dimensional connected compact non-Kähler, nearly Kähler manifold with constant scalar curvature. If $M$ satisfies the condition $T(\rho, \sigma)(\rho>0)$, and is holomorphically $\delta(>2 /(\rho+3))$ pinched, then $M$ is an Einstein space and the first Chern form of $M$ vanishes.

Proof. By the hypothesis and (2.10), (2.11), (2.24), we get

$$
\begin{aligned}
K(x, y) & \geqq(1 / 4)(3 \delta-2+3 \rho \delta) l \\
& >\frac{\rho}{\rho+3} l \quad(>0),
\end{aligned}
$$

for $x, y \in M_{p}$ with $\|x\|=\|y\|=1,\langle x, y\rangle=\langle x, J y\rangle=0$, for all $p \in M$. Thus, by (2.11), (2.25) and (4.17), we get

$$
\begin{aligned}
& K(x, y)+K(x, J y)+B(x, y) \\
& \quad=2\left\{K(x, y)+K(x, J y)-\left\|\left(\nabla_{x} J\right) y\right\|^{2}\right\} \\
& \quad \geqq(3 \delta-2) l+\left\|\left(\nabla_{x} J\right) y\right\|^{2} \\
& \quad \geqq\{(3+\rho) \delta-2\} l>0,
\end{aligned}
$$

and hence $M$ satisfies the condition (4.8) in Theorem 4.4. Thus, from Theorem 4.4 , it follows that

$$
\nabla R_{1}=0 \text { and } R_{1}=5 R_{1}^{*} .
$$

Thus, taking account of (2.4), (2.5) and (4.14), we may easily see that $M$ is an Einstein space.

Q.E.D.

In [17], Takamatsu and the second named author have proved the following 
Proposition 4.6. There does not exist any dimensional, except 6-dimensional, non-Kähler, nearly Kähler manifold of constant holomorphic sectıonal curvature.

From Propositions 4.1 and 4.6, it follows immediately that a non-Kähler, nearly Kähler manifold of constant holomorphic sectional curvature is a 6-dimensional space of positive constant curvature, and satisfies the condition $T(1,1)$. In the rest of this section, we shall prove a result (Theorem 4.10) related to Proposition 4.6. We assume that $M=(M, J,\langle\rangle$,$) is an n(=2 m)$-dimensional connected non-Kähler, Einstein nearly Kähler manifold with vanishing first Chern form, and furthermore satisfies the condition $T(\rho, \sigma)$ with $5 \rho>4 \sigma$ and is holomorphically $\delta(>2 /(\rho+3))$-pinched. First, we estimate the values of the functions $f_{\lambda}(\lambda=1,3,4)$ on $S(M)$.

LEMMA 4.7. For each point $(p, x) \in S(M)$, we have

$$
f_{1}(p, x) \geqq \frac{l}{8}\{(5 \rho-4 \sigma)(n+2) \delta-8 \rho\} H(x) .
$$

Proof. Let $\left\{e_{i}\right\}=\left\{e_{\alpha}, e_{\bar{\alpha}}\right\} \quad\left(x=e_{1}\right)$ be an orthonormal basis of $M_{p}$ which diagonalizes the matrix $\left(\left\langle\left(\nabla_{e_{i}} J\right) x,\left(\nabla_{e_{j}} J\right) x\right\rangle\right)(1 \leqq i, j \leqq n)$. Then, by the hypothesis for $M$ and (2.10), (2.11), (2.16), (3.13), (3.23) and (4.17), we get

$$
\begin{aligned}
f_{1}(p, x) & =\sum_{\imath, j} R_{\imath x \bar{j} \bar{x}}\left\langle\left(\nabla_{e_{i}} J\right) x,\left(\nabla_{e_{\jmath}} J\right) x\right\rangle \\
& =\sum_{\imath} R_{\imath x \imath x}\left\|\left(\nabla_{e_{i}} J\right) x\right\|^{2}-\sum_{\imath}\left\|\left(\nabla_{e_{i}} J\right) x\right\|^{4} \\
& \geqq \frac{S}{5 n}(5 \rho-4 \sigma) H(x)-\rho H(x)^{2} \\
& \geqq \frac{n+2}{8}(5 \rho-4 \sigma) \delta l H(x)-\rho l H(x) \\
& =\frac{l}{8}\{(5 \rho-4 \sigma)(n+2) \delta-8 \rho\} H(x) .
\end{aligned}
$$

LEMMA 4.8. For each point $(p, x) \in S(M)$, we have

$$
f_{3}(p, x) \leqq \delta l\left(\frac{S}{n}-H(x)\right) .
$$

Proof. Let $\left\{e_{i}\right\}=\left\{e_{\alpha}, e_{\bar{\alpha}}\right\}\left(x=e_{1}\right)$ be an orthonormal basis of $M_{p}$ as in the proof of Lemma 4.7. Then, by (2.10), (2.11), (3.13) and (4.17), we get

$$
\begin{aligned}
f_{3}(p, x) & =\sum_{\imath}\left\langle R\left(x,\left(\nabla_{e_{i}} J\right) x\right) x,\left(\nabla_{e_{i}} J\right) x\right\rangle \\
& \leqq \sigma H(x)\left(\frac{S}{n}-H(x)\right) \\
& \leqq \sigma l\left(\frac{S}{n}-H(x)\right) .
\end{aligned}
$$


LEMMA 4.9. For each point $(p, x) \in S(M)$, we have

$$
f_{4}(p, x) \leqq-\frac{n+2}{2} \delta l H(x) .
$$

Proof. By (2.10), (2.20), (3.13) and (3.23), we get

$$
\begin{aligned}
f_{4}(p, x) & =-\frac{4 S}{5 n} H(x) \\
& \leqq-\frac{n+2}{2} \delta l H(x) .
\end{aligned}
$$

Next, we estimate the value $\int_{S_{p}} g_{3} \omega_{2}$. By (2.10), (2.11), (3.16), (3.20), (3.21) and (3.22), we have

$$
\int_{S_{p}} g_{3} \omega_{2} \leqq \sigma l\left[\int_{S_{p}} \sum_{k}\left(R_{x \bar{x} x k}\right)^{2} \omega_{2}-\int_{S_{p}} H^{2} \omega_{2}\right]=\frac{\sigma l}{16} \int_{S_{p}}\left\|\operatorname{grad}^{v} H\right\|^{2} \omega_{2} .
$$

We are now in a position to prove the following

THEOREM 4.10. Let $M=(M, J,\langle\rangle$,$) be an n(\geqq 6)$-dimensional connected compact non-Kähler, nearly Kähler manifold with constant scalar curvature. If $M$ satisfies the condition $T(\rho, \sigma)$ with $5 \rho>4 \sigma, 3 \rho \geqq 4 \sigma-1$, and is holomorphically $\delta$-pinched $(\delta>2 /(\rho+3)$ and $\delta \geqq(4 \sigma+3 \rho) /(15 \rho-12 \sigma+4))$, then $M$ is isometric to $a$ 6-dimensional sphere of constant curvature.

Proof. First of all, we note

$$
1-\frac{4 \sigma+3 \rho}{15 \rho-12 \sigma+4}=\frac{4(3 \rho-4 \sigma+1)}{15 \rho-12 \sigma+4} \geqq 0
$$

and

$$
\begin{aligned}
& \frac{4 \sigma+3 \rho}{15 \rho-12 \sigma+4}-\frac{5(n+2) \sigma+24 \rho-8 \sigma}{(n+2)(15 \rho-12 \sigma+4)} \\
& =\frac{(n-6)(3 \rho-\sigma)}{(n+2)(15 \rho-12 \sigma+4)} \\
& \geqq \frac{(n-6)(3 \sigma-2 \rho)}{(n+2)(15 \rho-12 \sigma+4)} \geqq 0 .
\end{aligned}
$$

Next, from the hypothesis for $M$ and Theorem 4.5 , it follows that $M$ is an Einstein space with vanishing first Chern form. Furthermore, by (3.19), (4.18), (4.19) and Lemmas $4.7 \sim 4.9$, we have

$$
\begin{aligned}
& 0 \geqq 2 \int_{S(M)} g_{1} \omega \\
+ & \frac{l}{2}\left\{\frac{(n+2)(15 \rho-12 \sigma+4) \delta+8 \sigma-24 \rho-4(n+2) \sigma}{4(n+2)}+\frac{2 \rho}{\rho+3}\right\} \int_{S(M)}\left\|\operatorname{grad}^{v} H\right\|^{2} \omega
\end{aligned}
$$




$$
\begin{aligned}
& +\frac{32 S^{2} l}{25 n^{2}(n+2)^{2}}\{(15 \rho-12 \sigma+4)(n+2) \delta+8 \sigma-24 \rho-5(n+2) \sigma\} V_{2} \operatorname{Vol}(M) \\
\geqq & 2 \int_{S(M)} g_{1} \omega+\frac{l}{2}\left(\frac{\sigma}{4}+\frac{2 \rho}{\rho+3}\right) \int_{S(M)}\left\|\operatorname{grad}^{v} H\right\|^{2} \omega \geqq 0 .
\end{aligned}
$$

Thus, from (4.20) and Proposition 3.2, it follows that $M$ is a space of constant holomorphic sectional curvature. Therefore, the theorem follows immediately from Propositions 4.1 and 4.6.

Q.E.D.

\section{§. An example.}

We shall recall some elementary facts about Riemannian 3-symmetric spaces (cf. [8], [21]). Let $(G / K, J,\langle\rangle$,$) be a compact Riemannian 3-symmetric space$ such that the Riemannian metric $\langle$,$\rangle is determined by a biinvariant Riemannian$ metric on $G$ and $J$ is the canonical almost complex structure. Then it is known that $(G / K, J,\langle\rangle$,$) is a nearly Kähler manifold ([8]). We denote by g$ and $f$ the Lie algebras of $G$ and $K$ respectively. Then we have the following direct sum decomposition $([8])$ :

$$
\mathfrak{g}=\mathfrak{f}+\mathfrak{m}, \quad \operatorname{Ad}(K) \mathfrak{m}=\mathfrak{m},
$$

where $\mathfrak{m}$ is the orthogonal complement of $\mathfrak{f}$ in $g$. We may identify the subspace $\mathfrak{m}$ with the tangent space $(G / K)_{e K}$ of $G / K$ at the origin $e K \in G / K$. Under this identification, we have the following formulas ([8], [20]):

$$
\begin{aligned}
& \left(\nabla_{x} J\right) y=-J[x, y]_{\mathfrak{m}}, \quad x, y \in \mathfrak{m}, \\
& K(x, y)=\frac{1}{4}\left\|[x, y]_{\mathfrak{m}}\right\|^{2}+\left\|[x, y]_{\mathfrak{t}}\right\|^{2}, \\
& x, y \in \mathfrak{m} \quad \text { with }\|x\|=\|y\|=1,\langle x, y\rangle=0 .
\end{aligned}
$$

In particular, we consider the 6-dimensional compact Riemannian 3-symmetric space $(S p(2) /(U(1) \times S p(1)), J,\langle\rangle$,$) in which the Riemannian metric \langle$,$\rangle is induced$ from the inner product

$$
(x, y)=- \text { Real part of }(\text { Trace } x y), \quad x, y \in \mathfrak{s p}(2) .
$$

We put $G=S p(2)$ and $K=U(1) \times S p(1)$. Let $\boldsymbol{H}$ be the algebra of quaternions, i.e.,

$$
\begin{gathered}
\boldsymbol{H}=\left\{q=a_{0}+a_{1} \boldsymbol{e}_{1}+a_{2} \boldsymbol{e}_{2}+a_{3} \boldsymbol{e}_{3} \mid a_{0}, a_{1}, a_{2}, a_{3} \in \boldsymbol{R}, \quad \boldsymbol{e}_{i}^{2}=-1(1 \leqq i \leqq 3),\right. \\
\left.\boldsymbol{e}_{1} \boldsymbol{e}_{2}=-\boldsymbol{e}_{2} \boldsymbol{e}_{1}=\boldsymbol{e}_{3}, \quad \boldsymbol{e}_{2} \boldsymbol{e}_{3}=-\boldsymbol{e}_{3} \boldsymbol{e}_{2}=\boldsymbol{e}_{1}, \quad \boldsymbol{e}_{3} \boldsymbol{e}_{1}=-\boldsymbol{e}_{1} \boldsymbol{e}_{3}=\boldsymbol{e}_{2}\right\}
\end{gathered}
$$

Then it is well known that the Lie algebra $\mathfrak{a p}(2)$ of $S p(2)$ is given by

$$
\mathfrak{s} \mathfrak{p}(2)=\left\{\left.x \in \mathfrak{g} \mathfrak{l}(2, \boldsymbol{H})\right|^{t} x=-\bar{x}\right\} .
$$

We put 


$$
\begin{array}{ll}
x_{1}=\frac{1}{\sqrt{2}}\left[\begin{array}{rr}
0 & 1 \\
-1 & 0
\end{array}\right], & y_{1}=\frac{1}{\sqrt{2}}\left[\begin{array}{cc}
0 & \boldsymbol{e}_{1} \\
\boldsymbol{e}_{1} & 0
\end{array}\right], \\
x_{2}=\left[\begin{array}{cc}
-\boldsymbol{e}_{2} & 0 \\
0 & 0
\end{array}\right], & y_{2}=\left[\begin{array}{cc}
\boldsymbol{e}_{3} & 0 \\
0 & 0
\end{array}\right], \\
x_{3}=\frac{1}{\sqrt{2}}\left[\begin{array}{cc}
0 & \boldsymbol{e}_{2} \\
\boldsymbol{e}_{2} & 0
\end{array}\right], & y_{3}=\frac{1}{\sqrt{2}}\left[\begin{array}{cc}
0 & \boldsymbol{e}_{3} \\
\boldsymbol{e}_{3} & 0
\end{array}\right], \\
s_{1}=\left[\begin{array}{cc}
0 & 0 \\
0 & \boldsymbol{e}_{2}
\end{array}\right], & s_{2}=\left[\begin{array}{ll}
0 & 0 \\
0 & \boldsymbol{e}_{3}
\end{array}\right], \\
t_{1}=\left[\begin{array}{ll}
\boldsymbol{e}_{1} & 0 \\
0 & 0
\end{array}\right], & t_{2}=\left[\begin{array}{ll}
0 & 0 \\
0 & \boldsymbol{e}_{1}
\end{array}\right] .
\end{array}
$$

Then we see that $\left\{x_{2}, y_{2}(1 \leqq i \leqq 3), s_{1}, s_{2}, t_{1}, t_{2}\right\}$ is an orthonormal basis of $\mathfrak{g}=\mathfrak{g p}(2)$ and the Lie algebra $\mathfrak{f}$ of $K$ (resp. the subspace $\mathfrak{m}$ of $\mathfrak{g}$ in the decomposition (5.1)) is linearly spanned by $\left\{s_{1}, s_{2}, t_{1}, t_{2}\right\}$ (resp. $\left\{x_{\imath}, y_{\imath}(1 \leqq i \leqq 3)\right\}$ ) over $\boldsymbol{R}$ (cf. [15]).

The canonical almost complex structure $J$ is given by

$$
J x_{\imath}=y_{\imath}, \quad J y_{\imath}=-x_{\imath} \quad(1 \leqq i \leqq 3) .
$$

By (5.2), we get

$$
\left\|\left(\nabla_{x} J\right) y\right\|^{2}=1,
$$

for $x, y \in \mathfrak{m}$ with $\|x\|=\|y\|=1,\langle x, y\rangle=\langle x, J y\rangle=0$. By (5.6), we see that $(S p(2) /(U(1) \times S p(1)), J,\langle\rangle$,$) is a non-Kähler, nearly Kähler manifold.$

By (5.3), (5.4) and (5.5), by direct computation, we get

$$
\begin{aligned}
H(x) & =\left\|[x, J x]_{\mathbf{x}}\right\|^{2} \\
& =2\left\{5\left(a_{1}^{2}+b_{1}^{2}+a_{3}^{2}+b_{3}^{2}-\frac{3}{5}\right)^{2}+\frac{1}{5}\right\},
\end{aligned}
$$

for any unit vector $x=a_{1} x_{1}+b_{1} y_{1}+a_{2} x_{2}+b_{2} y_{2}+a_{3} x_{3}+b_{3} y_{3} \in \mathfrak{m}$. By (5.7), we have easily

$$
\frac{2}{5} \leqq H(x) \leqq 4
$$

Thus, by (5.6) and (5.8), we see that $(S p(2) /(U(1) \times S p(1)), J,\langle\rangle$,$) is holomorph-$ ically $1 / 10$-pinched and satisfies the condition $T(1 / 4,5 / 2)$.

Let $x$ be any unit vector in $\mathfrak{m}$ and $y$ any unit vector in $\mathfrak{m}$ which is orthogonal to $x$. Then we may put

$$
y=a J x+b z,
$$

where $z$ is a unit vector in $\mathfrak{m}$ with $\langle x, z\rangle=0,\langle J x, z\rangle=0$, and $a, b \in \boldsymbol{R}$ with $a^{2}+b^{2}=1$. By (5.3), taking account of (5.2), (5.6) and (5.9), we have 


$$
\begin{aligned}
K(x, y) & =\frac{1}{4}\left\|b[x, z]_{\mathfrak{m}}\right\|^{2}+\left\|a[x, J x]_{\mathfrak{t}}+b[x, z]_{\mathfrak{l}}\right\|^{2} \\
& =\frac{b^{2}}{4}+\left\|a[x, J x]_{\mathfrak{z}}+b[x, z]_{\mathfrak{l}}\right\|^{2} .
\end{aligned}
$$

Therefore, by (5.7), (5.8) and (5.10), we may easily see that $(S p(2) /(U(1) \times S p(1))$, $J,\langle\rangle$,$) has strictly positive sectional curvature.$

We remark that $S p(2) /(U(1) \times S p(1))$ is diffeomorphic to a complex projective space of complex dimension 3 ([15]). We also note that $\mathrm{K}$. Furukawa has obtained the estimation (5.8) in unpublished work.

\section{REFERENCES}

[1] BERger, M., Les variétés riemanniennes homogénes simplement connexes à courbure strictement positive, Ann. Scuola Norm. Sup. Pisa, 15 (1961), 179-246.

[2] Berger, M., Gauduchon, P. and E. Mazet, Le spectre d'une variété riemannienne, Lecture Notes in Math. 194, Springer (1971).

[3] Bishop, R.L. AND S.I. Goldberg, On the second cohomology group of a Kähler manifold of positive curvature, Proc. Amer. Math. Soc. 16 (1965), 119-122.

[4] BISHOP, R.L. AND S.I. GoldBERG, Rigidity of positively curved Kähler manifolds, Proc. Nat. Acad. Sci. 54 (1965), 1037-1041.

[5] Bishop, R.L. AND S.I. Goldberg, On the topology of positively curved Kähler manifolds II, Tôhoku Math. J. 17 (1965), 310-318.

[6] Furukawa, K. and S. Sawaki, Pinching of a 6-dimensional nearly Kähler manifold, Tensor, N.S., 38 (1982), 53-61.

[7] GRAY, A., Nearly Kähler manifolds, J. Differential Geometry 4 (1970), 283-309.

[8] Gray, A., Riemannian manifolds with geodesic symmetries of order 3, J. Differential Geometry 7 (1972), 343-369.

[9] Gray, A., The structure of nearly Kähler manifolds, Math. Ann. 223 (1976), 233-248.

[10] GraY, A., Compact Kähler manifolds with nonnegative sectional curvature, Inventiones Math. 41 (1977), 33-43.

[11] Matsumoto, M., On 6-dimensional almost Tachibana spaces, Tensor, N.S., 23 (1972), 250-252.

[12] SASAKI, S., On the differential geometry of tangent bundles of Riemannian manifolds, Tôhoku Math. J. 10 (1958), 338-354.

[13] Sato, T., Riemannian 3-symmetric spaces and homogeneous $K$-spaces, Memoirs Fac. Tech., Kanazawa Univ., 12 (1979), 137-143.

[14] Sekigawa, K., Almost Hermitian manifolds satisfying some curvature conditions, Kodai Math. J. 2 (1979), 384-405.

[15] Sekigawa, K. and J. Watanabe, On some compact Riemannian 3-symmetric spaces, Sci. Rep. Niigata Univ. 19 (1983), 1-17.

[16] TaChibana, S., On infinitesimal conformal and projective transformations of compact $K$-spaces, Tôhoku Math. J. 13 (1961), 386-392.

[17] Takamatsu, K. And T. Sato, A $K$-space of constant holomorphic sectional curvature, Kodai Math. Sem. Rep. 27 (1976), 116-127.

[18] Tanno, S., Constancy of holomorphic sectional curvature in almost Hermitian 
manifolds, Kodai Math. Sem. Rep. 25 (1973), 190-201.

[19] Tricerri, F. AND L. VANHECKe, Curvature tensors on almost Hermitian manifolds, Trans. Amer. Math. Soc. 267 (1981), 365-398.

[20] Wallach, N.R., Compact homogeneous Riemannian manifolds with strictly positive curvature, Ann. of Math. 96 (1972), 277-295.

[21] Wolf, J.A. AND A. GRAY, Homogeneous spaces defined by Lie group automorphisms, I, II, J. Differential Geometry 2 (1968), 77-159.

[22] Yano, K. AND S. Bochner, Curvature and Betti numbers, Annals of Math. Studies 32, Princeton Univ. Press, 1953.

$\begin{array}{ll}\text { Nilgata University } & \text { Kanazawa University } \\ \text { Nilgata, Japan } & \text { KanaZawa, Japan }\end{array}$

